\title{
Digitalisierung als Gewinnerin der Corona-Krise. Und das EPD?
}

\section{Yvonne Gilli}

Dr. med., Mitglied des FMH-Zentralvorstandes, Departementsverantwortliche Digitalisierung/eHealth

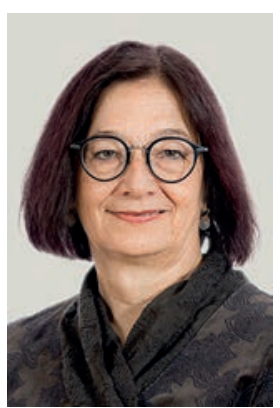

Im April 2020 beschrieb die NZZ die digitale Welt als «die Gewinnerin der Corona-Krise» [1]. Und tatsächlich zeigt sich in der momentanen Situation das Potenzial digitaler Hilfsmittel: Mittels Videokonsultationen konnte die Gesundheitsversorgung an vielen Orten unterstützt werden, und mit der Proximity Tracing App ${ }^{1}$ des Bundes steht eine digitale Form des Contact Tracing zur Verfügung. Mit Letzterem hat die Schweiz innert kürzester Zeit beispiellos eine verglichen mit anderen europäischen Ländern führende Rolle eingenommen. Über das bisher grösste Digitalisierungsprojekt im Gesundheitswesen - das elektronische Patientendossier (EPD) - sucht man seit Beginn der Pandemie jedoch vergebens Schlagzeilen. Neben der COVID-19-dominierten Berichterstattung hängt dies auch damit zusammen, dass die Einführung des EPD, statt wie geplant diesen Frühling, erst gegen Ende des Jahres erfolgen wird. Der Hauptgrund dafür liegt nicht in der COVID-19-Pandemie, sondern bei der Zertifizierung der (Stamm-)Gemeinschaften: Diese Organisationen werden das EPD schlussendlich anbieten und müssen daher von einer privaten Zertifizierungsstelle hinsichtlich ihrer Konformität zum Bundesgesetz über das EPD geprüft werden. Und die Zertifizierung ist komplexer als lange angenommen.

Auch wenn Ende Jahr die Zertifizierungen abgeschlossen sind, wird das EPD nicht zum Selbstläufer - zu komplex ist das Grossprojekt. Prinzipiell haben Grossprojekte im Gesundheitswesen mit den gleichen Schwierigkeiten zu kämpfen wie diejenigen anderer Branchen und unterliegen ebenso der Gesetzmässigkeit des Scheiterns. ${ }^{2}$ Sie unterscheiden sich jedoch in einigen wesentlichen Punkten: Die Umsetzung von IT-Projekten im Gesundheitswesen verlangt klar definierte Standards für Interoperabilität und Terminologien neben den gesetzlich einzuhaltenden Anforderungen. Darüber hinaus müssen die Systeme flexibel genug sein, um verschiedene Organisationen zu unterstützen - von kleinen Einzelpraxen angefangen bis hin zu grossen integrierten Versorgungsnetzwerken. Im Idealfall verbessern diese Systeme auch Arbeitsabläufe, senken Kosten und verbessern die Qualität der Versorgung, während gleichzeitig die seit langem bestehenden vorteilhaften Muster der Kommunikation und Zusammenarbeit bestehen bleiben.
Ein integraler Bestandteil des Erfolgs in der Umsetzung von nationalen Grossprojekten wie dem EPD ist stets eine Mischung aus organisatorischen, verhaltensbezogenen, kognitiven und sozialen Faktoren [2]. Es muss frühzeitig festgestellt werden, wer «Erfolg» definiert und wann «Erfolg» bestimmt wird. Dieser misst sich anhand verschiedener Dimensionen; unter anderem an der Verbesserung von Informations- und Systemqualität, der Verbreitung oder Auswirkung auf individueller und organisatorischer Ebene [3]. Im Falle des EPDs ist offensichtlich, dass sich in der jetzigen Phase der Erfolg primär an der technischen Umsetzung und der Anzahl zertifizierter Stammgemeinschaften misst.

Auf der individuellen Ebene entstehen für den einzelnen Arzt bei der Nutzung des EPDs ganz andere Fragestellungen: Wie bewährt sich der Zugriff auf das EPD mit einer Zwei-Faktor-Authentifizierung in einer Arztpraxis oder in einem Spital zwischen Bett- und Raumund Patientenwechsel? Wie sind die relevanten Informationen im EPD möglichst rasch auffindbar? Anfang Juni fand zur Klärung dieser und anderer Fragen eine erste Sitzung der neuen «FMH-Arbeitsgruppe EPD» statt - einer Gruppe von ärztlichen Vertretern aus kantonalen Ärztegesellschaften sowie von Ärztinnen und Ärzten, die mit den (Stamm-)Gemeinschaften gut vernetzt sind. Ziel dieser Arbeitsgruppe ist die Koordination der kantonalen ärztlichen Tätigkeiten im Bereich des EPDs sowie die Erarbeitung von gemeinsamen Positionen und Vorschlägen zur Lösung von entstehenden Herausforderungen im Rahmen der EPD-Einführung. Einmal mehr zeigt sich: Die digitale Welt kann nur gewinnen, wenn sie geprägt wird von Professionalität und von der Erfahrung der betroffenen Fachpersonen. In diesem Fall sind dies die Ärztinnen und Ärzte.

\section{Literatur}

1 Simanowski R. Die Corona-Krise pflügt unsere Gesellschaft um und wenn es einen Gewinner gibt, dann ist es die digitale Welt. Neue Zürcher Zeitung [Internet]. 2020 Apr 20; Available from: https://www.nzz.ch/meinung/es-gibt-einen-sieger-in-der-coronakrise-die-digitale-welt-ld.1551064

2 Kaplan B, Harris-Salamone KD. Health IT Success and Failure: Recommendations from Literature and an AMIA Workshop. J Am Med Informatics Assoc. 2009.

3 Zozus MN, Penning M, Hammond WE. Factors impacting physician use of information charted by others. JAMIA Open. 2019. 